\title{
Longer term visual outcome of eyes with retinopathy of prematurity treated with cryotherapy or diode laser
}

\author{
M O’Keefe, J O’Reilly, B Lanigan
}

\begin{abstract}
Aims-Visual outcome of 66 eyes in 37 patients who had undergone treatment with either cryotherapy or diode laser for threshold retinopathy of prematurity was assessed.

Methods-17 patients, representing 30 eyes treated with cryotherapy, were examined at between 56 and 98 months corrected age (median 68 months). 20 patients representing 36 eyes treated with diode laser, were examined at between 30 and 66 months corrected age (median 51 months). Structural outcome was categorised as: optimal-flat posterior pole; suboptimal-macular ectopia, optic nerve hypoplasia, retinal fold involving the macula, and retinal detachment involving the macula.

Results-Optimal structural outcome was, in the absence of amblyopia, associated with optimal visual acuity (of $6 / 12$ or better) in all cases, with most eyes achieving a visual acuity of $6 / 9$ or $6 / 6$. Suboptimal structural outcome was invariably associated with suboptimal visual acuity. Amblyopia was present in eight out of 20 cryotherapy treated eyes and in five out of 26 laser treated eyes with an optimal structural outcome. Refractive errors were significantly less in laser treated eyes as was the incidence of anisometropic amblyopia.

Conclusion-Eyes treated with either cryotherapy or diode laser for threshold retinopathy of prematurity with optimal structural outcome are associated with development of optimal visual acuitythat is, $6 / 12$ or better. Treatment with either cryotherapy or laser does not in itself reduce the visual potential of these eyes.

(Br f Ophthalmol 1998;82:1246-1248)
\end{abstract}

Children's Hospital, Temple Street, Dublin, Ireland

M O'Keefe

J O'Reilly

B Lanigan

Correspondence to: Mr Michael O'Keefe, The Children's Hospital, Temple Street, Dublin 1, Ireland.

Accepted for publication 15 April 1998 retinopathy (Cryo-ROP) was the first study to demonstrate conclusively that treatment for threshold (stage $3+$ ), retinopathy of prematurity (ROP) was beneficial. ${ }^{1-3}$ However, assessment of functional outcome at $31 / 2$ and $51 / 2$ years revealed that although cryotherapy greatly reduced the incidence of poor visual outcomes, it did not increase the number of eyes that achieved normal visual acuity of $6 / 12$ or more. ${ }^{4}$ This was explained by a large number of eyes that achieved an intermediate visual acuity of between $6 / 60$ and 6/12.
In the years since the CRYO-ROP study was designed and carried out, laser therapy has become an established mode of treatment. Although no randomised trials have been performed, several studies have shown laser treatment to be at least as effective as cryotherapy in inducing regression of threshold $\mathrm{ROP}^{6-11}$

In this study we examined the visual status of the eyes that we had treated with either cryotherapy or diode laser.

\section{Methods}

Between January 1986 and November 199133 eyes in 19 patients who survived the neonatal period, were treated with cryotherapy (Cryo group) for threshold ROP (defined as 5 or more contiguous clock hours, 30 sectors, or 8 cumulative clock hours of stage $3+$ disease in zone 1 or 2). Gestational age ranged from 25 to 30 weeks, with a mean of 26.8 weeks. Birth weight ranged from $685 \mathrm{~g}$ to $1500 \mathrm{~g}$ (mean 949 g). Between December 1991 and September 1994, 41 eyes in 23 patients (laser group) who survived the neonatal period, were treated with diode laser for threshold ROP. Gestational age ranged from 24 to 32 weeks with a mean of 26.7 weeks. Birth weight ranged from $685 \mathrm{~g}$ to $1360 \mathrm{~g}$ with a mean of $779 \mathrm{~g}$. Infants treated after the end of September 1994 were excluded from the study as they would have been under the age of 30 months at the time of follow up (April 1997).

The indications for treatment and the methods of treatment employed in each of these groups have been described in detail elsewhere and did not differ in any significant way from standard techniques. ${ }^{6} 12$ Treatment with cryotherapy was performed under a general anaesthetic. A double ring of cryotherapy burns was applied to the avascular retina anterior to the fibrovascular ridge in the first treatment session. A small number of cases required additional treatment to induce regression. Laser treatment was performed under sedation and involved the placement of burns anterior to the fibrovascular ridge. Subsequent therapy

Table 1 Methods employed for visual acuity assessment in eyes with quantifiable vision

\begin{tabular}{lcc}
\hline $\begin{array}{l}\text { Method of visual acuity } \\
\text { assessment }\end{array}$ & $\begin{array}{l}\text { Cryo group } \\
\text { (No of eyes) }\end{array}$ & $\begin{array}{l}\text { Laser group } \\
\text { (No of eyes) }\end{array}$ \\
\hline Snellen & 18 & 4 \\
Crowded letter testing & 2 & 2 \\
Sheridan-Gardiner & 2 & 11 \\
Allen picture cards & 1 & 12 \\
Teller acuity cards & 0 & 2 \\
\hline
\end{tabular}


Table 2 Overall structural and functional outcome of eyes treated with cryotherapy and of eyes treated with laser

\begin{tabular}{|c|c|c|c|c|c|c|c|c|c|c|c|c|c|}
\hline \multirow{2}{*}{$\begin{array}{l}\text { Structural } \\
\text { outcome }\end{array}$} & \multicolumn{5}{|c|}{ Cryo group: visual outcome } & \multirow{2}{*}{$\begin{array}{l}\text { Totals } \\
(n=30 \\
\text { eyes })\end{array}$} & \multicolumn{6}{|c|}{ Laser group: visual outcome } & \multirow{2}{*}{$\begin{array}{l}\text { Totals } \\
(n=36 \\
\text { eyes) }\end{array}$} \\
\hline & $6 / 12-6 / 6$ & $6 / 12-6 / 18$ & $6 / 18-6 / 60$ & $<6 / 60$ & Blind & & $6 / 12-6 / 6$ & $6 / 12-6 / 18$ & $6 / 18-6 / 60$ & $<6 / 60$ & Blind & Unrecordable & \\
\hline Optimal & 16 & 3 & 1 & 0 & 0 & 20 & 23 & 3 & 0 & 0 & 0 & 0 & 26 \\
\hline Macular ectopia & 0 & 1 & 0 & 0 & 0 & 1 & 0 & 0 & 1 & 0 & 0 & 0 & 1 \\
\hline $\mathrm{ONH}$ & 0 & 0 & 1 & 0 & 0 & 1 & 0 & 0 & 2 & 0 & 0 & 2 & 4 \\
\hline Tractional fold & 0 & 0 & 0 & 1 & 0 & 1 & 0 & 0 & 0 & 2 & 0 & 0 & 2 \\
\hline Poor & 0 & 0 & 0 & 0 & 7 & 7 & 0 & 0 & 0 & 0 & 3 & 0 & 3 \\
\hline Totals & 16 & 4 & 2 & 1 & 7 & 30 & 23 & 3 & 3 & 2 & 3 & 2 & 36 \\
\hline
\end{tabular}

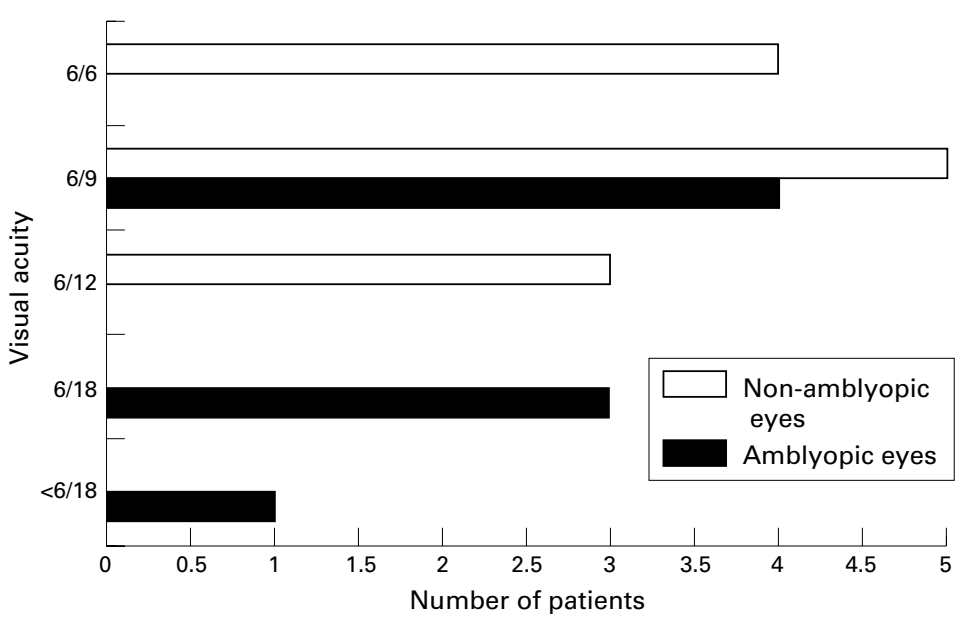

Figure 1 Visual acuity in amblyopic and non-amblyopic eyes, with an optimal structural outcome, after treatment with cryotherapy.

consisted of a fill in pattern of burns in the gaps between those previously applied.

Fifty months or more of follow up was obtained on 17 patients in the cryotherapy group; 13 of these patients had undergone bilateral treatment and four had undergone unilateral treatment representing 30 treated eyes. The remaining two patients died before the age of 30 months. Age at follow up ranged between 56 and 98 months with a median of 68 months.

Twenty patients in the laser group were followed up for 30 months or more; 16 of these patients had undergone bilateral treatment and four had undergone unilateral treatment representing 36 treated eyes. One patient died before the age of 30 months and two patients were lost to follow up. Age at follow up ranged between 30 and 66 months with a median of 51 months. Structural outcome was graded as follows:

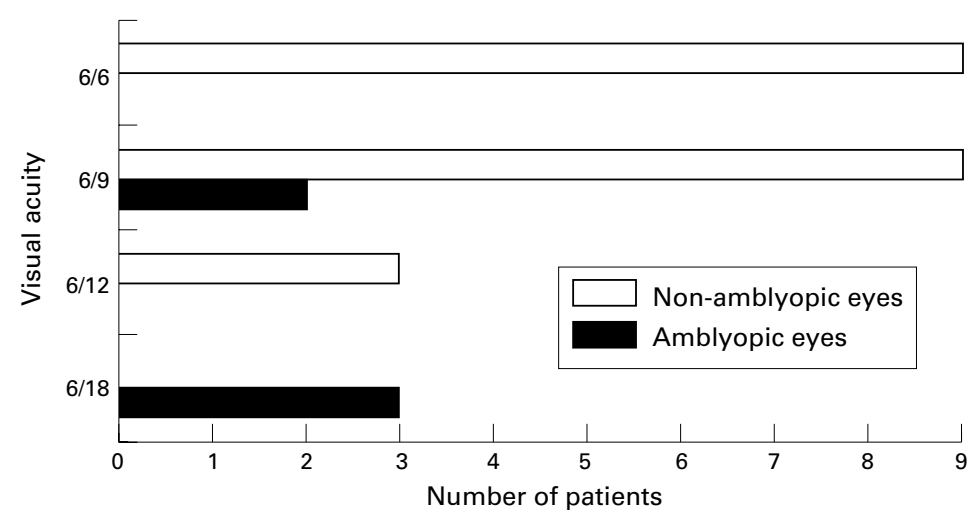

Figure 2 Visual acuity in amblyopic and non-amblyopic eyes, with an optimal structural outcome, after treatment with diode laser.
Optimal: flat posterior pole, with a normal optic disc and uncompromised macula.

Suboptimal included macular ectopia, flat posterior pole in the presence of pale hypoplastic optic discs associated with grade 3-4 IVH and hydrocephalus.

Retinal folds involving the macula, associated with a flat posterior pole and detached macula with or without peripheral retinal detachment.

Eyes were assigned to the appropriate categories on the basis of fundal observations made by one observer only (MO'K).

All visual assessments were performed by either an experienced orthoptist or a senior ophthalmic nurse. Methods employed for visual assessment varied according to the age and mental ability of each child. Details of the methods used are shown in Table 1 .

\section{Results}

Over two third of eyes in both groups achieved optimal structural outcomes (Table 2). The majority of these eyes achieved a visual acuity of $6 / 12$ or better at final follow up, with most achieving a visual acuity of $6 / 6$ or $6 / 9$ (Figs 1 and 2). Suboptimal structural outcome as a result of macular ectopia or optic nerve hypoplasia $(\mathrm{ONH})$ was also associated with intermediate visual acuity at the final follow up visit. Visual acuity was unrecordable in one patient with $\mathrm{ONH}$ who was severely mentally handicapped. Retinal folds involving the macula resulted in visual acuity of less than $6 / 60$ in every case, while retinal detachments involving the macula resulted in a visual acuity ranging between hand movements and no perception of light.

Overall, six eyes in the cryo group and six eyes in the laser group achieved a visual acuity of less than $6 / 12$ but more than 6/60. Suboptimal structural outcomes (that is, macular ectopia and optic nerve hypoplasia), accounted for two eyes in the cryo group and three eyes in the laser group. Amblyopia (four eyes in the cryo group and three eyes in the laser group), accounted for intermediate visual acuity recorded in the remaining eyes. All amblyopic eyes achieved good visual acuities in the contralateral non-amblyopic eyes.

Eight out of 20 eyes with an optimal structural outcome developed amblyopia (five strabismic, three anisometropic), in the cryo group as did five out of 26 eyes in the laser treated group (all as a result of strabismus). All amblyopic patients were treated with patching of the contralateral eye and many of these eyes achieved vision of $6 / 12$ or better. 
Refractive outcome was considerably better in the laser treated eyes. Of 26 eyes with an optimal structural outcome in the laser treated group, 13 eyes had normal refractive errors not requiring correction. The remaining 13 eyes all had refractive errors of less than 5.0 DS. Two of 20 eyes with optimal structural outcome, in the cryotherapy group, did not require correction: eight had refractive errors or less than 5.0 DS, while 10 had refractive errors of more than, or equal to 5.0 DS (all myopic).

\section{Discussion}

It is now over 10 years since cryotherapy was established by the multicentre Cryo-ROP study as an established treatment for ROP. As a result, the identification of threshold disease offers a narrow window in which treatment is most likely to be effective, The only change in this regard is the introduction of laser. Several studies, albeit uncontrolled, have shown it to be at least as effective as cryotherapy in inducing regression of ROP. ${ }^{6-11}$ However, much of the poor visual outcome is the result of poor retinal structural outcome despite timely and proper treatment. ${ }^{12}$ Could earlier treatment improve the structural outcome in some children? This has to be weighed against possible surgical complications and unnecessary treatment. Flynn has suggested that future advances in treatment will be dependent on the classification of disease morphology and more appropriate use of treatment modalities in order to reduce the overall incidence of poor structural outcome. ${ }^{13}$

The results of the multicentre trial of cryotherapy for retinopathy of prematurity at $31 / 2$ and 5 years demonstrated that while cryotherapy greatly reduced the incidence of poor visual outcome, it did not greatly increase the number of eyes that achieved a normal visual acuity of $6 / 12$ or better. ${ }^{14}$ Therefore, the finding that a large number of eyes achieved a good structural outcome but only "intermediate" visual acuity was suggestive of the fact that cryotherapy might have reduced the potential for vision in treated eyes.

We recognise the limitations of our study are many; it is not controlled, the two groups are historically separated, and the numbers are small. While we have tried as far as possible to apply the same criteria of treatment in both groups, this is clearly open to observer error. As a general observation, however, although the international classification has served us well in terms of treatment, it is in itself open to observer error in the documentation of the location and extent of disease.

The longer term follow up documents an improved refractive outcome with the laser treated group and this has been seen in other studies. ${ }^{15} 16$ The finding of an improved refractive outcome may explain the absence of anisometropic amblyopia in the laser treated group. We recognise, however, that the methods of visual assessment in some of the laser treated group are open to more favourable visual interpretation because of their age.

In conclusion, since the Cryo-ROP study no other large well designed clinical trial has emerged to challenge some of our current practice in the management of this condition and most of the studies are from single centres, with small numbers of patients, and uncontrolled. However, while recognising this fact, it is reassuring that the longer term results of eyes treated with either cryotherapy or laser with good structural outcome have a good visual outcome. Treatment with either method does not in itself appear to reduce the visual potential in these eyes.

1 Cryotherapy for Retinopathy of Prematurity Cooperative Group. Multicentre trial of cryotherapy for retinopathy of prematurity. Pre

2 Cryotherapy for Retinopathy of Prematurity Cooperative Group. Multicentre trial of cryotherapy for retinopathy of prematurity. Three month outcome. Arch Ophthalmol 1990;108:195-204.

3 Cryotherapy for Retinopathy of Prematurity Cooperative Group. Multicentre trial of cryotherapy for retinopathy of prematurity. One year outcome-structure and function. Arch Ophthalmol 1990;108:1408-16.

4 Cryotherapy for Retinopathy of Prematurity Cooperative Group. Multicentre trial of cryotherapy for retinopathy of prematurity. Three and a half-year outcome-structure and function. Arch Ophthalmol 1993;111:339-44.

5 Cryotherapy for Retinopathy of Prematurity Cooperative Group. Multicentre trial of cryotherapy for retinopathy of prematurity. Snellen visual acuity and structural outcome at five and a half years after randomization. Arch Ophthalmol 1996;114:417-424.

6 Goggin M, O'Keefe M. Diode laser for retinopathy of prematurity-early outcome. Br f Ophthalmol 1993;77: $559-62$

7 McNamara JA, Tasman W, Vander JF, et al. Diode laser photocoagulation for retinopathy of prematurity. Preliminary results. Arch Ophthalmol 1992;110:1714-6.

8 Capone A, Diaz-Rohena R, Sternberg P, et al. Diode laser photocoagulation for zone 1 threshold retinopathy of prematurity. Am f Ophthalmol 1993;116:444-50.

9 The Laser ROP Study Group. Laser therapy for retinopathy of prematurity. Arch Ophthalmol 1994;112:154-6.

10 Ling CS, Fleck BW, Wright E, et al. Diode laser treatment for retinopathy of prematurity:structural and functional for retinopathy of prematurity:structural and

11 White JE, Repka MX. Randomised comparison of diode laser photocoagulation versus cryotherapy for threshold retinopathy of prematurity: 3 year outcome. $\mathcal{F}$ Paediatr Ophthalmol Strabismus 1997;34:83-7.

12 Knight-Nanan DM, Algawi K, Bowell R, et al. Advanced cicatricial retinopathy of prematurity-outcome, complications. Brf Ophthalmol 1996;80:343-5.

13 Flynn JT. Retinopathy of prematurity:perspective for the nineties. Acta Ophthalmol Scand 1995:(Suppl) 214:12-14.

14 Reynolds J, Dobson V, Quinn GE, et al for the Cryotherapy for Retinopathy of Prematurity Cooperative Group. Prediction of visual function in eyes with mild to moderate posterior pole residua of retinopathy of prematurity. Arch Ophthalmol 1993;111:1050-6.

5 Knight DM, O'Keefe M. Refractive outcome in eyes with retinopathy of prematurity treated with cryotherapy or diode laser: 3 year follow up. Br F Ophthalmol 1996;80:9981001.

16 Laws F. Laws D, Clark D. Cryotherapy and laser treatment for acute retinopathy of prematurity: refractive outcomes, a longitudinal study. Br f Ophthalmol 1997;81:12-15. 